\title{
$\alpha$-乳糖水溶液太赫兹吸收光谱实验研究及模拟分析 ${ }^{*}$
}

\author{
侯磊王俊喃王否施卫 \\ (西安理工大学应用物理系, 西安 710048)
}

\section{摘 要}

水对太赫兹 $(\mathrm{THz})$ 波有强烈的吸收, 所以利用 $\mathrm{THz}$ 技术研究含水样 品特性一直是本领域的难题。本文在 $\mathrm{THz}$ 时域光谱系统中通过使用工 作于高倍增模式的光电导天线和喇叭形渐变平行平板波导提高了样品 所在位置处的 $\mathrm{THz}$ 波电场强度, 实现了在 $0.1-1.5 \mathrm{THz}$ 范围对 $\alpha$ - 孚糖溶 液 $\mathrm{THz}$ 吸收光谱的直接检测。进而, 运用密度泛函理论奶水㺷境下 $\alpha$ 乳糖单分子模型的 $\mathrm{THz}$ 吸收光谱进行模拟仿真研究、模拟结果与实验 测试结果吻合较好。本文对直接检测含水样品在 THz 波段的光谱特性 具有重要参考价值。

关键词: 太赫兹波, 太赫兹时域光谱系统, 密度泛函理论, $\alpha$-乳糖

PACS: $32.30 . \mathrm{Bv}, \quad 31.15 . \mathrm{E}^{-}, \quad 33.20 . \mathrm{Tp}$

基金: 国家自然科学基金（批准号：62075179，61575161), 陕西省自然科学基金重点 项目 (批准号: 2019JZ-04), 陕西省超快光电与太赫兹科学重点实验室（批准号: 2018SZS-06); 西安市超快光电器件技术重点实验室（批准号：201805055ZD6CG39） 资助的课题.

$\dagger$ 通讯作者. E-mai1. Swshi@mail. xaut. edu. cn

第一作者, E-mai 1: houleixaut@126.com

\section{1 引言/}

太赫兹波（Terahertz wave, THz wave）是频率在 0.1 到 $10 \mathrm{THz}$, 处于远红 外和微波之间的一段电磁辐射 ${ }^{[1]}$, 具有瞬态性、相干性、宽带性、穿透性、低 能性等很多优异的性能, 在分子生物学 ${ }^{[2,3]}$ 、医药学 ${ }^{[4-6]}$ 等诸多领域具有广阔的 应用前景。特别是由于 $\mathrm{THz}$ 波的光子能量只有毫电子伏特量级, 不会对生物组 织产生有害的光电离, 与大多数有机分子的振动和转动能级相当, 表现出特有 
的指纹谱, 因此利用 $\mathrm{THz}$ 波检测有机分子具有独特的优势。

乳糖（Lactose, $\mathrm{C}_{12} \mathrm{H}_{22} \mathrm{O}_{11}$ ) 是 $\mathrm{D}$-葡萄糖与 $\mathrm{D}$-半乳糖以 $\beta-1,4$ 键结合的二糖, 它作为人类和哺乳动物乳汁中特有的碳水化合物, 是维持人和动物生命所需的 主要能源物质。乳糖有 $\alpha$-乳糖和 $\beta$-乳糖两种异构体, $\alpha$-乳糖易与一分子结晶水 结合, 变为 $\alpha$-乳糖一水合物, 所以乳糖共有 $\alpha$-乳糖一水合物、 $\alpha$-乳糖无水物和 $\beta$-乳糖三种构型。目前, 国内外已经有多个关于利用太赫兹时域光谱 (Terahertz time-domain spectroscopy, THz-TDS）技术研究固态乳糖样品的报道。2007 年, E. R. Brown 等人 ${ }^{[7]}$ 实验测得乳糖一水合物在 $0.53 \mathrm{THz}$ 处出现窝带强吸收, 通过 高分辨率光谱技术和洛伦兹函数拟合确定了其半峰全宽为 $23 \mathrm{GHz}$ 。2013 年, A. I. Mcintosh 等人 ${ }^{[8]}$ 利用 THz-TDS 系统研究了高湿度条件下的乳糖结晶, 获得了 有关结晶速率及其动力学的数据。2015 赵国忠等人 ${ }^{[9]}$ 实验得到 4 种乳糖在 0.2-2.6 THz 范围内的吸收谱并模拟分析得出吸收峰主要来源于分子内或者分子 间的氢键振动。同年, 鹿文亮等人 ${ }^{[10]}$ 对乳糖和氨基苯甲酸在 $0.1-1 \mathrm{THz}$ 波段内 进行了伪彩 $\mathrm{THz}$ 成像实验, 通过两种物质的 $\mathrm{THz}$ 图像的颜色差别来区分两种物 质及其分布, 并得到乳糖在 $0.53 \mathrm{THz}$ 的吸收峰。2019 年, 陈涛等人 ${ }^{[11]}$ 利用 THz-TDS 系统得到 乳糖一水合物和 D-葡萄糖在 0.3-1.7 THz 频段的特征吸收 峰并进行了理论分析, 结果表明 $\mathrm{THz}$ 波对结构相似的单糖和二糖的结构差异十 分敏感。2020 年, Y. M. Wang 等人 ${ }^{[12]}$ 实验测得 L-组氨酸在 $0.77 \mathrm{THz}$ 和 $\alpha$-乳糖 在 $0.53 \mathrm{THz}$ 的特征吸收峰并利用特征吸收峰实现了对两种物质的快速指认。上 述研究结果表明, THz 波在有机物的检测与识别方面有其独特的优势, 然而由 于水对 $\mathrm{THz}$ 波的强烈吸收 ${ }^{[13]}$, 检测含水样品及生物样品的 $\mathrm{THz}$ 吸收特性非常困 难。为了实现对含水样品的 $\mathrm{THz}$ 检测, 2015 年, $\mathrm{M}$ Grognot 等人 ${ }^{[14]}$ 使用 $\mathrm{THz}$ 时 
域衰减全反射（ATR）技术实现了对上皮细胞的无损检测, 但是 ATR 系统比较 复杂。2018 年, K. L. Shig 等人 ${ }^{[15]}$ 利用微流控芯片测得了 THz 波在三种不同浓 度酒精溶液和三磷酸腺苷溶液中的透过率, 但是微流控芯片的测量灵敏度会因 为所使用液体量的减少而降低。2019 年, A Keshavarz 等人 ${ }^{[16]}$ 利用超材料传感 器对 H1N1、H5N2 和 H9N2 三种病毒进行了测试, 得出了三种病毒分别在 $1.668 \mathrm{THz} 、 1.665 \mathrm{THz}$ 和 $1.641 \mathrm{THz}$ 处有特征吸收峰。但是超材料学般只对特定 频率表现出较高的 $\mathrm{Q}$ 值, 并且测试结果会受到其表面样品均匀性的影响。

本文提出了一种操作简便、准确度高的对含水样品进行直接检测的方法。 利用光电导天线的高倍增模式提高了 $\mathrm{THz}$ 波的辐射功率 使用喇叭形渐变平行 平板波导 (HSTPPW) 进一步增强 THz 电场, 从而实现对乳糖水溶液 $\mathrm{THz}$ 光谱 信息的直接检测。为了进一步验证实验测得的乳糖水溶液的 $\mathrm{THz}$ 光谱信息并分 析其与分子结构之间的关系，运角密度泛函理论（Density Functional Theory, DFT) 对水环境下 $\alpha$-乳糖一水合物单分子模型进行了模拟仿真研究, 根据仿真 结果对实验结果在 0.1-1.5 5 Hz 范围内特征峰的振动模式进行了分析和归属。

\section{2 高倍增 $\mathrm{THz}$ 光車导天线}

由于水对 $\mathrm{THz}$ 波有强烈的吸收, 因此提高 $\mathrm{THz}$ 源的辐射功率是有效检测含 水样品在 $\mathrm{THz}$ 波段的吸收特性的关键。光电导天线是一种常用的 $\mathrm{THz}$ 辐射源, 通常情况下, 光电导天线工作于线性工作模式, 即 1 个光子至多产生 1 个电子 空穴对, 这些电子和空穴在偏置电场的作用下加速运动, 产生 $\mathrm{THz}$ 辐射, 由于 电子质量小, 它对 $\mathrm{THz}$ 辐射起主要作用。GaAs 光电导天线在满足一定的电场 阈值和光能阈值的条件下可以工作在高倍增模式, 此时 GaAs 材料吸收光子后, 产生的电子在强偏置电场作用下加速运动, 通过级联碰撞过程使载流子的数目 
发生雪崩倍增, 此时 1 个光子可等效产生 $10^{3}-10^{5}$ 个电子空穴对, 因此辐射 $\mathrm{THz}$ 波的功率可大幅提高。但是, 由于高倍增模式下电流存在锁定效应, 在此阶段 天线不会辐射 $\mathrm{THz}$ 波, 并且由于电流的热效应还会对天线的耐压和寿命带来不 利影响, 因此需要在光激发电荷畴形成后破坏其维持条件导致其迅速猝灭, 天 线关断, 获得具有载流子倍增效应但是不具有锁定效应的电流脉冲, 从而使光 电导天线辐射出高功率 $\mathrm{THz}$ 波。实验中, 使用的光电导天线间隙为 $0.927 \mathrm{~mm}$, 当偏置电压为 $3100 \mathrm{~V}$ 时, 光电导天线工作于线性模式和高倍增模式的临界状态; 偏置电压为 $3200 \mathrm{~V}$ 时，光电导天线出现明显的高倍增现象 1 图 1 是偏置电压为 $3100 \mathrm{~V}$ 和 $3200 \mathrm{~V}$ 时，天线在线性模式和高倍增模式 辐射 $\mathrm{THz}$ 波的时域波形。 此时, 两种工作模式的偏置电压仅差 $100 \mathrm{~V}$, 高倍增模式下天线辐射 $\mathrm{THz}$ 波的 电场强度的峰峰值比线性模式提高了 4.4 倍 对应的 $\mathrm{THz}$ 波功率提高了 19.36 倍。

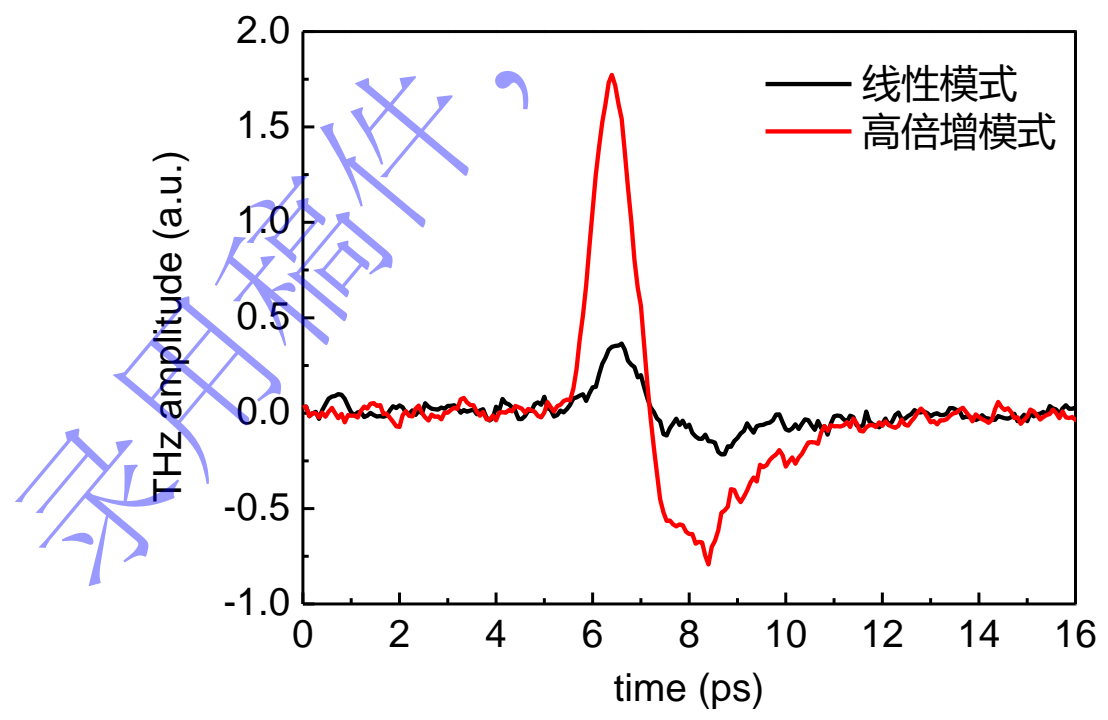

图 $1 \mathrm{GaAs}$ 光电导天线在线性模式和高倍增模式辐射 $\mathrm{THz}$ 波的时域波形

Fig.1 THz time domain waveforms radiated by GaAs photoconductive antenna in linear mode and high multiplication mode. 


\section{3 实验}

\section{1 实验装置}

含水样品测试系统是一种带有 HSTPPW 的 THz-TDS 系统, 光路示意图如 图 2（a）所示。采用飞秒激光器 (Spectra-Physics) 作为光源, 激光波长为 $800 \mathrm{~nm}$, 重复频率为 $1 \mathrm{kHz}$, 平均功率为 $5 \mathrm{~W}$, 脉宽为 $100 \mathrm{fs}$ 。飞秒激光束经过 一个 $70 / 30$ 分束器 (BS) 后分为两束, 较强的作为葲浦光, 较弱的作为探测光。 泵浦光束经过由多层介质膜反射镜 M2, M3, M4 组成的延迟系统后被焦距为 $100 \mathrm{~mm}$ 的透镜（L1）聚焦在光电导天线上。天线中产生光生载流子, 在两端所 加载的偏置电场作用下加速运动辐射 $\mathrm{THz}$ 波。产生的 THz 波通过焦距为 4 英寸 的离轴抛物面镜 (PM1) 准直后耦合到 HSTPPW 曲, HSTPPW 的间隙为 $100 \mu \mathrm{m}$, 待测样品放置于 HSTPPW 的中心, 如图 2 (b) 所示。 THz 波在波导内传输过 程中, 样品厚度很薄, 较强的 THz 电场可以穿透样品, 因此从 HSTPPW 出射 的 $\mathrm{THz}$ 波携带了样品信息。 $\mathrm{THz}$ 波被另一个焦距为 4 英寸的离轴抛物面镜 (PM2) 收集汇聚, 经过氧化铟锡（ITO）玻璃反射聚焦到 ZnTe 晶体上, 引起 $\mathrm{ZnTe}$ 的偏振态改变。探测光经反射镜 M5、M6 反射后由透镜（L2）聚焦在 $\mathrm{ZnTe}$ 晶体上, 透射光携带了 $\mathrm{THz}$ 波的信息。探测光束经过 $1 / 4$ 波片（QWP）, 透镜（L3）和渥拉斯顿棱镜（Wollaston Prism）后分成偏振方向相互垂直的两 束光, 汇聚平平衡探测器的两个探头上, 经过差分运算后的信号输入锁相放大 器。该系统最大的特点是, 使用了高倍增工作模式的光电导天线和 HSTPPW 增 强了样品所在位置处 $\mathrm{THz}$ 波的电场强度。 


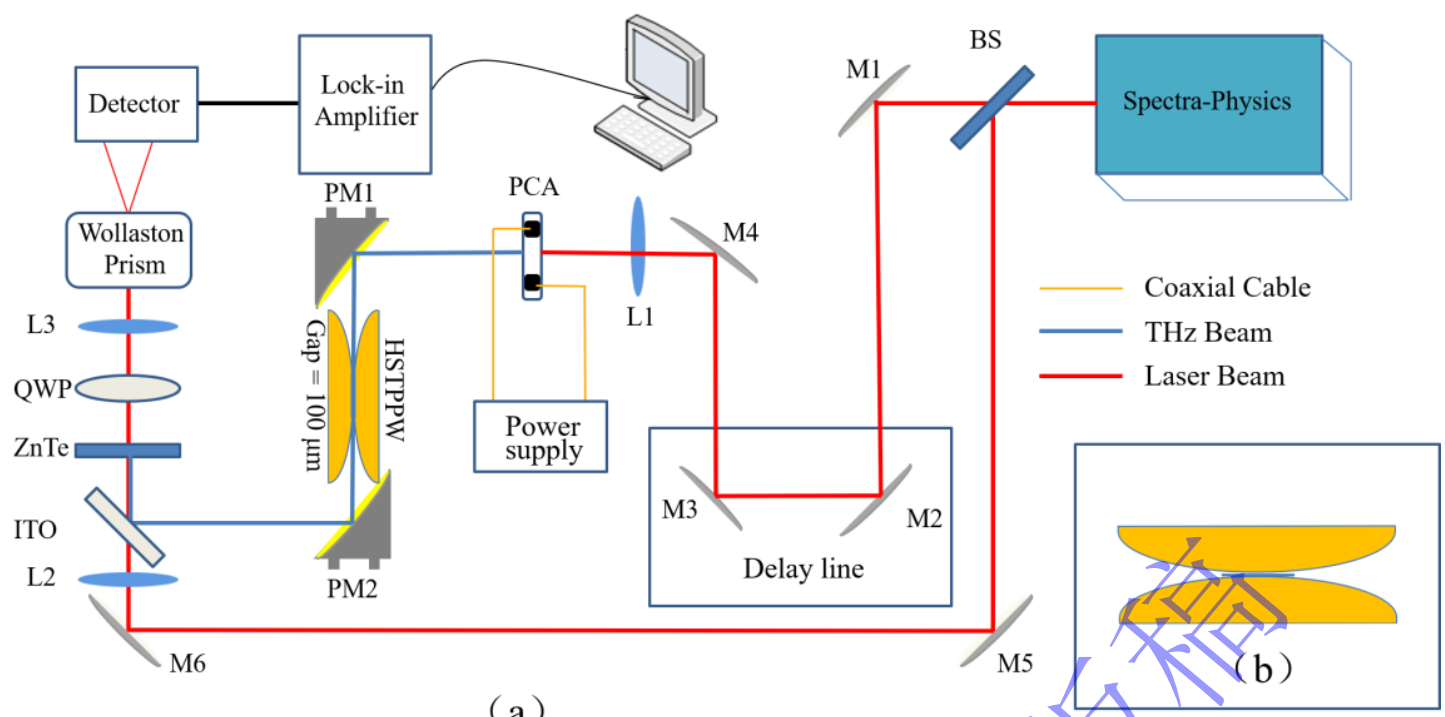

(a)

图 2（a）带有喇叭形渐变平行平板波导的 THz-TDS 系统; (b) 喇叭形渐变平行平板波导 结构示意图

Fig. 2. (a) Schematic of homemade THz-TDS system/with/a horn shaped tapered parallel plate waveguide; (b) The structural diagram of horn shaped tapered parallel plate wave.

\section{2 实验及结果分析}

本实验中所使用的 $\alpha$-乳糖一水合物样品购自中国天津市红岩化学试剂厂, 将 $\alpha$-乳糖一水合物溶解在去离子水中制备浓度为 $5 \times 10^{-4} \%$ 乳糖稀溶液。由于水 在 $\mathrm{THz}$ 波段也有吸收峰, ’因此为了减少水的吸收峰对乳糖吸收峰的影响, 我们 用去离子水作为参考。用移液枪将 $20 \mu \mathrm{L}$ 纯净水滴入 HSTPPW 下半片的中心位 置, 放1 严片, 中间形成 $100 \mu \mathrm{m}$ 厚的水膜, 利用 THz-TDS 系统检测 $\mathrm{THz}$ 波 经过水后的时域波形, 经傅里叶变换得到频谱, 作为参考。把水擦干, 用移液 枪 取 $20 \mu \mathrm{L} \alpha$-乳糖水溶液滴到 HSTPPW 的中心位置, 用同样的方法形成 100 $\mu \mathrm{m}$ 厚的溶液膜, 测试乳糖水溶液的 $\mathrm{THz}$ 时域波形和频谱。将乳糖水溶液的 $\mathrm{THz}$ 谱除以去离子水的 $\mathrm{THz}$ 谱获得乳糖水溶液在 $\mathrm{THz}$ 波段的特征波形。

室温情况下, 对于频率为 $1 \mathrm{THz}$ 的 $\mathrm{THz}$ 波经过厚度为 $d$ 的水膜后, 出射光 
子数 $N_{o u t}$ 和入射光子数 $N_{i n}$ 的关系式 (1) 计算。

$$
N_{\text {out }}=e^{-\alpha d} N_{\text {in }},
$$

其中, 水的吸收系数 $\alpha=220 \mathrm{~cm}^{-1}[17]$ 。当 $d=100 \mu \mathrm{m}$ 时, 由式 (1) 计算可得每 有 9 个太赫兹光子入射, 有 1 个太赫兹光子出射。文中增强了 $\mathrm{THz}$ 电场强度, 增加了入射含水样品的 $\mathrm{THz}$ 光子数目, 从而实现利用 $\mathrm{THz}$ 波对含水样品的直接 探测。

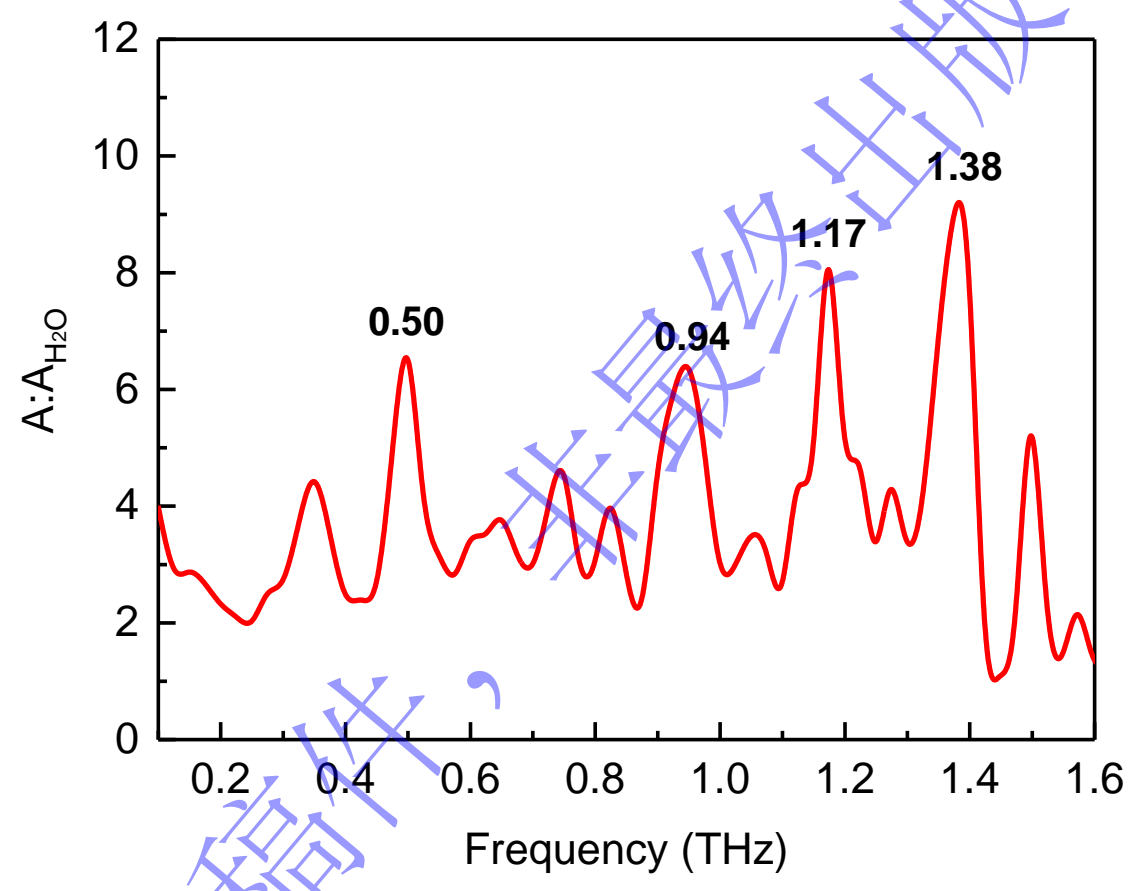

图 $3 \alpha$-乳糖水溶液的 $\mathrm{THz}$ 吸收光谱

Fig. 3. THz absorption spectrum for $\alpha$-lactose aqueous solutions, which was measured by the homemade THz-TDS system with parallel planar waveguide.

图 3 是通过实验测试获得的 $\alpha$-乳糖水溶液在 0.1-1.6 THz 范围内的吸收光谱 曲线。从图中知在 $0.50 、 1.17 、 1.38 \mathrm{THz}$ 处存在三个吸收峰, 与参考文献[8]中 报道的 $0.53 \mathrm{THz}, 1.20 \mathrm{THz}, 1.38 \mathrm{THz}$; 参考文献[11]中报道的 $0.53 \mathrm{THz}, 1.19$ $\mathrm{THz}, 1.38 \mathrm{THz}$; 参考文献[18]中报道的 $0.53 \mathrm{THz}, 1.20 \mathrm{THz}, 1.38 \mathrm{THz}$ 吸收峰 
相吻合。但是, 由于本文测试的是乳糖水溶液, 而文献 $[8,11,17]$ 测试的是固态 乳糖, 因此上述数据不可能完全一致。一方面是由于所使用测试仪器的频谱分 辨率导致的, 另一方面是在水环境下受到水中氢键的影响乳糖的分子结构与固 态时不同。

虽然我们使用水的信号作为参考信号, 但是纯水与 $\alpha$-乳糖水溶液中水分子 的特性也不完全相同, 采用水的信号作为参考信号可以明显减弱 0 -糖乳糖水溶 液中大多数水峰的影响, 从而使 $\alpha$-乳糖的吸收峰凸显出来, 但是不能完全消除 水峰的影响, 因此还会在图中看到水的吸收峰, 如 $0.35 \mathrm{THz}, 0.64 \mathrm{THz} 、 1.04$ $\mathrm{THz}$ 和 $1.49 \mathrm{THz}$ 。图 3 中其他的峰可能是由于 $\alpha$-乳糖在水溶液中产生端基异构 体 $\beta$-糖乳糖的吸收峰, 以及溶液中水和乳糖形成的新的化学键形成的吸收峰。

\section{4 数值模拟及结果分析}

\section{1 分子模型与计算方法}

为了进一步分析实验结果, 了解乳糖水溶液在 $\mathrm{THz}$ 波段吸收峰的产生机理, 利用 Gaussian16 软件对 $\alpha$-乳糖一水合物单分子模型在水环境下的特性进行了仿 真模拟。单个 $\alpha$-乳糖 水合物分子结构模型从剑桥数据库系统 ${ }^{[19]}$ (Cambridge structural database system, CCDC) 中提取, 如图 4 所示。计算时选用包含梯 度修正的泛函 $\mathrm{B} 3 \mathrm{LY} \mathrm{P}^{[20,21]}$, 基组选用 6-31G(d), 同时为了减小在计算单个分子 时 $\mathrm{DFT}$ 不能很好的模拟范德瓦耳斯力而引起的误差 ${ }^{[22]}$, 引入 Grimme $^{[23]}$ 提出的 色散校正项 (B3LYP-D3BJ)。考虑到实验中样品处于水溶剂的情况, 模拟研究 中使用隐式溶剂模型, 这种溶剂模型不具体描述溶质附近的溶剂分子的具体结 构和分布, 而是把溶剂环境简单的当成可极化的连续介质来考虑, 使用隐式溶 剂模型可以在计算中表现出溶剂的平均效应。采用隐式溶剂模型中的 SMD 溶 
剂模型, 以水为溶剂。计算结果没有出现虚频, 说明对 $\alpha$-乳糖一水合物分子几 何优化至分子势能面极小点。

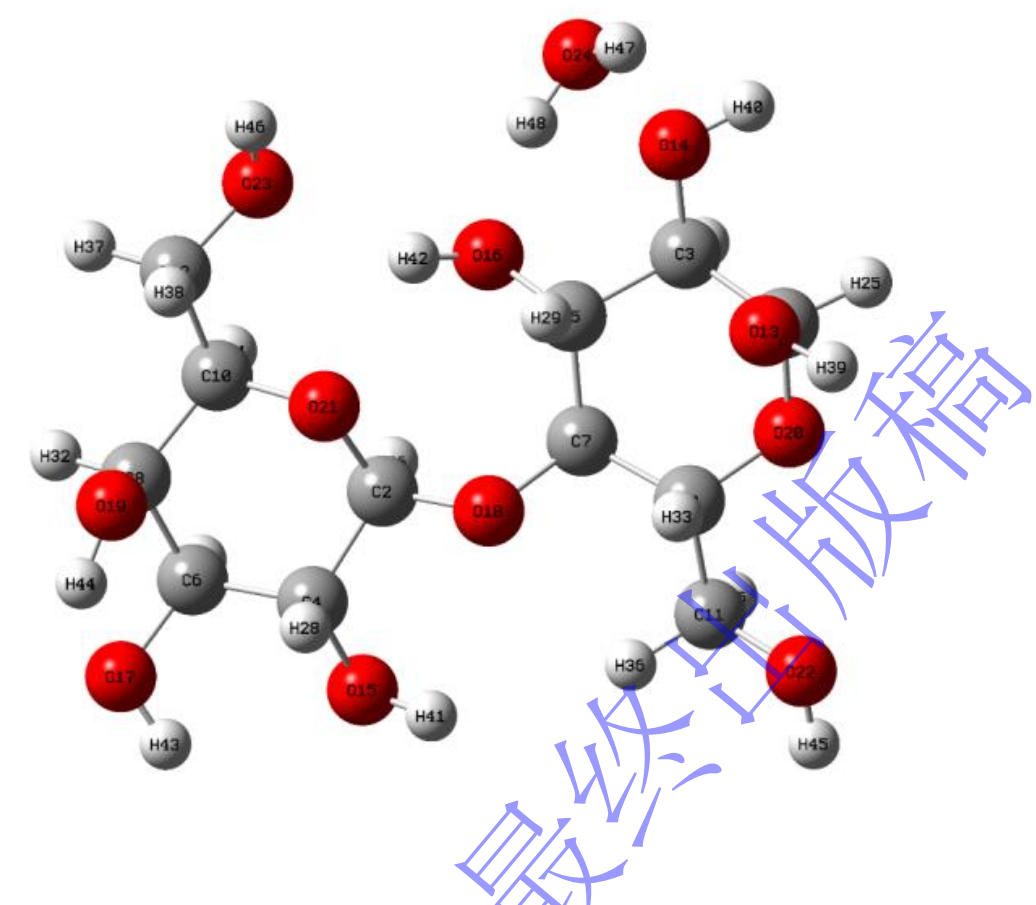

图 $4 \alpha$-乳糖飞水合物分子结构

Fig. 4. The molecular of $\alpha$-lactose monohydrate.

\section{2 振动分析}

结合计算结果的输出女件和 GaussView6.0 可以得到 $\alpha$-乳糖一水合物水溶液 在不同频率下的振动模武。图 5 展示了 $\alpha$-乳糖一水合物在 $0.55 \mathrm{THz} 、 0.98 \mathrm{THz}$ 、 $1.16 \mathrm{THz} 、 1.43 \mathrm{THz}$ 峰值所对应的振动模式, 图中蓝色箭头表示原子振动方向。
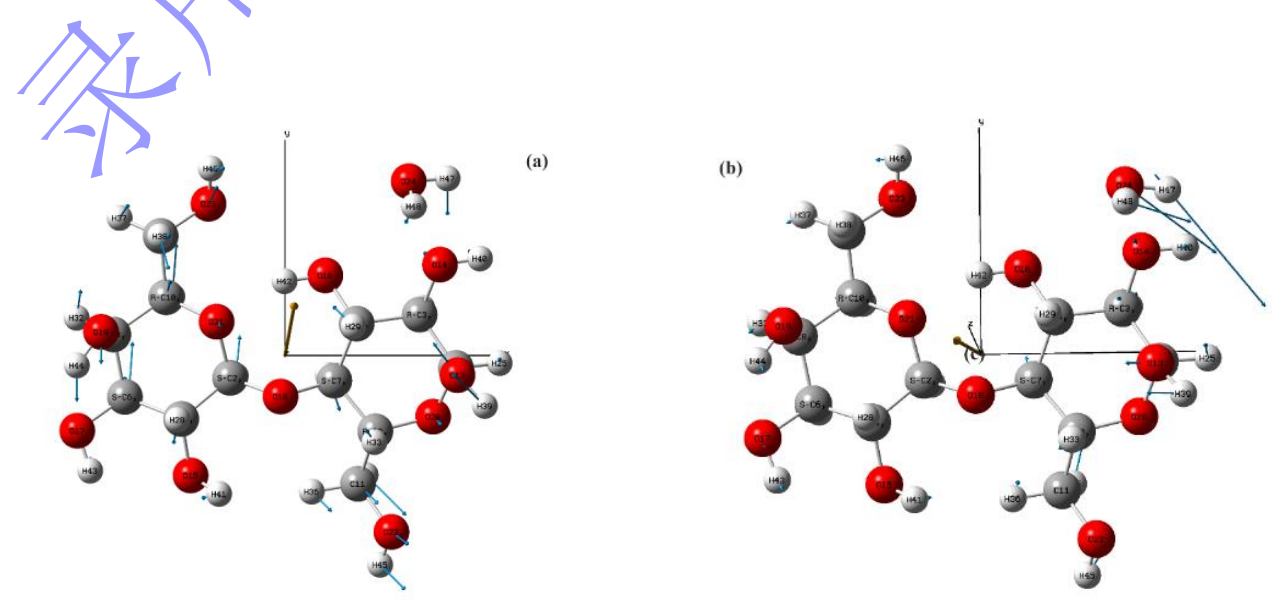

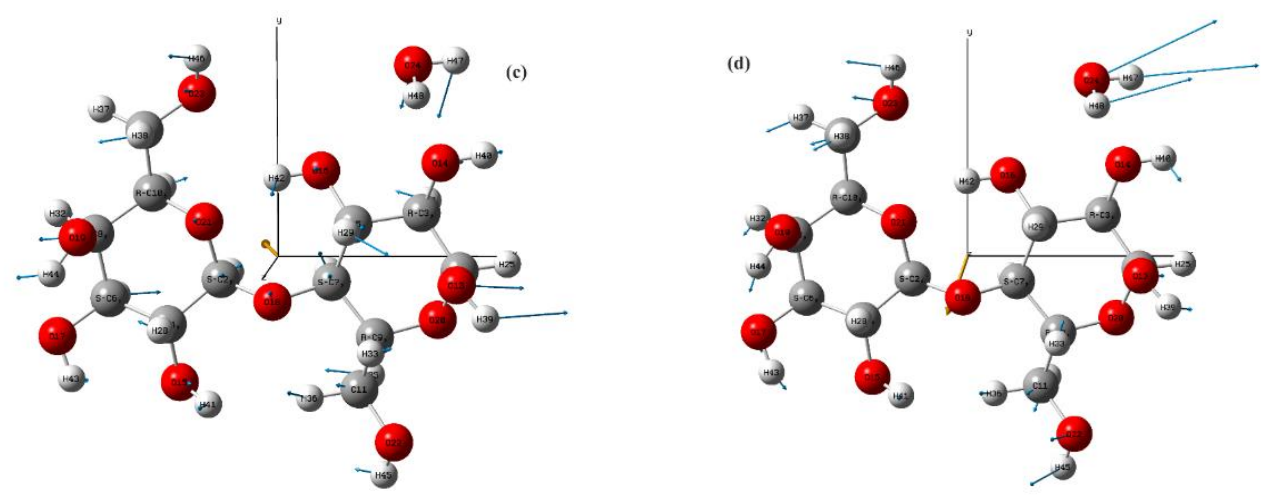

图 5 乳糖的振动模式 (a) $0.58 \mathrm{THz}$; (b) $1.01 \mathrm{THz}$ (c) $1.19 \mathrm{THz}$; (d) $1.47 \mathrm{THz}$

Fig. 5. Vibrational modes of $\alpha$-lactose monohydrate at (a) $0.58 \mathrm{THz} ;(\mathrm{b}) 1 . \overline{0} 1 \mathrm{THz} ;(\mathrm{c}) 1.19 \mathrm{THz}$;(d) $1.47 \mathrm{THz}$.

分子在 $\mathrm{THz}$ 频段的振动主要是源于多个原子参与的变形振动、扭绞振动和 弯曲振动 ${ }^{[24]}$ 。为了定性解释模拟结果, 通过 Gauss View6.0 和输出文件对 $\alpha$-乳糖 一水合物的振动模式进行简正振动（Potentia1 Energy Distributions, PED）分析, 得到不同频率下主要基团和原子振动模式对简正振动的贡献百分比, 结果见表 1 。 $\alpha$-乳糖一水合物在 $0.58 \mathrm{THz}$ 处的吸收峰主要是 C2-O18-C7 带动 C1-C3-C5C7-C9-O20 和 C2-C4-C6-e8-C10-O21 两个六圆环做反对称扭绞振动, 同时带动 C3-C5-C7-C9 与之连接的羟基和氢原子做面内摇摆振动; $1.01 \mathrm{THz}$ 处是以 $\mathrm{C} 2-$ O18-C7 带动 C1-C3-C5-C7-C9-O20 和 C2-C4-C6-C8-C10-O21 两个六圆环做剪式 振动, 同时带动水分子做面内弯曲振动; $1.19 \mathrm{THz}$ 处是以 $\mathrm{C} 2-\mathrm{O} 18-\mathrm{C} 7$ 带动 $\mathrm{C} 1-$ C3-C5-C7-C9-O20 和 C2-C4-C6-C8-C10-O21 两个六圆环做对称扭绞振动, C1C3-C5-C9 与之连接的着基和氢原子做扭绞振动同时带动水分子做面内弯曲振动; $1.47 \mathrm{THz}$ 处 C2-O18-C7 带动 C1-C3-C5-C7-C9-O20 和 C2-C4-C6-C8-C10-O21 两 个六圆环做弯曲振动, 同时 $\mathrm{C} 13$ 上的羟基带动水分子做弯曲振动。 
表 1 乳糖一水合物振动频率的 PED 分析

Table 1. Assignments of vibration frequencies for lactose monohydrate by PED.

\begin{tabular}{ccc}
\hline 物质 & 频率 & 振动模式 \\
\hline & $0.58 \mathrm{THz}$ & $\mathrm{C} 2-\mathrm{O} 18-\mathrm{C} 7 \& \mathrm{C} 3-\mathrm{C} 5-\mathrm{C} 7-\mathrm{C} 9(35.5 \%)$ \\
$\boldsymbol{\alpha}$-乳糖一水合物 & $1.01 \mathrm{THz}$ & $\mathrm{C} 2-\mathrm{O} 18-\mathrm{C} 7 \& \mathrm{C} 1-\mathrm{C} 3-\mathrm{C} 5-\mathrm{C} 7(34.5 \%)$ \\
& $1.19 \mathrm{THz}$ & $\mathrm{C} 2-\mathrm{O} 18-\mathrm{C} 7 \& \mathrm{C} 1-\mathrm{C} 3-\mathrm{C} 5-\mathrm{C} 7(44.6 \%)$ \\
& $1.47 \mathrm{THz}$ & $\mathrm{C} 2-\mathrm{O} 18-\mathrm{C} 7 \& \mathrm{C} 1-\mathrm{C} 3-\mathrm{C} 5-\mathrm{C} 7(35,7 \%)$ \\
\hline
\end{tabular}

\section{3 结果分析}

$\alpha$-乳糖一水合物水溶液的模拟结果如图 6 所示。在 $0.1-1.6 \mathrm{THz}$ 范围内有 $0.58 \mathrm{THz} 、 1.01 \mathrm{THz} 、 1.19 \mathrm{THz} 、 1.47 \mathrm{THz}$ 四余特征峰, 分别与实验结果中的 $0.50 \mathrm{THz}, 0.94 \mathrm{THz}, 1.17 \mathrm{THz}$ 和 $1.38 \mathrm{TH}$ 四个特征峰相对应。根据 3.2 中的分 析知, $1.01 \mathrm{THz}$ 处的特征峰与溶液中的承分子有关, 因此在之前的文献报道中, 乳糖固体的 $\mathrm{THz}$ 吸收光谱中此处没有吸收峰。

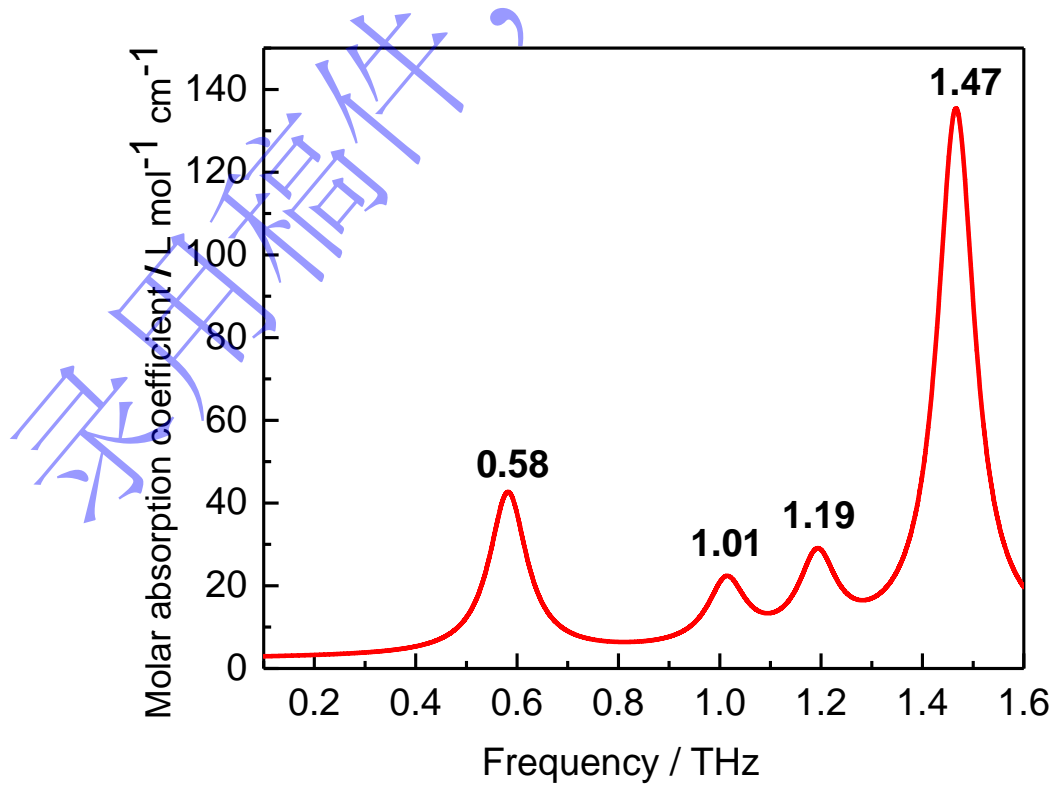

图 $6 \alpha$-乳糖一水合物水溶液计算光谱图

Fig. 6. Theoretical spectra for $\alpha$-lactose monohydrate. 
仿真结果和实验结果吻合较好, 说明实验所测结果是乳糖溶液的吸收峰, 也说明了所建仿真模型是合理的。但是, 实验结果和仿真结果还出现了一些偏 差, 其原因如下：第一, 与实际实验中的多分子体系不同，仿真中采用的是 $\alpha-$ 乳糖一水合物的单分子模型, 计算中没有考虑分子间的相互作用。第二, 仿真 模拟中使用的隐式溶剂模型也会引起误差, 该模型无法表现出溶剂和溶质之间 氢键等近程强相互作用，而且隐式溶剂模型还会改变体系的势能面，从而影响 到体系的几何优化和振动频率的计算。

\section{5 结论}

针对利用 $\mathrm{THz}$ 技术检测含水样品的难题, 本文利用非线性模式的光电导天 线产生高功率 THz 波, 并利用 HSTPPW 进韭增强 $\mathrm{THz}$ 电场的场强, 从而检 测得到了 $\alpha$-乳糖的水溶液在 0.1-1.5 THz 范围内的特征吸收峰, 分别为 $0.50 \mathrm{THz}$ 、 $0.94 \mathrm{THz} 、 1.17 \mathrm{THz}$ 和 $1.38 \mathrm{THz}$ 。运角密度泛函理论对水环境下的 $\alpha$-乳糖一水 合物进行仿真模拟, 并得到了与实验结果吻合度较高的仿真结果。通过 GaussView6.0 和计算输出学件对 $0.58 \mathrm{THz} 、 1.01 \mathrm{THz} 、 1.19 \mathrm{THz}$ 和 $1.47 \mathrm{THz}$ 太 赫兹吸收峰的振动模武进行了归属和分析, 较好地解释了该有机物太赫兹光谱 的形成机理。本文为研究含水物质在 $\mathrm{THz}$ 波段的特性提供了实验手段和分析方 法。

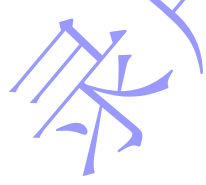




\section{参考文献}

[ 1 ] Ferguson B, Zhang X C 2002 Nat. Mater. 126

[2] Menikh A, Michkan P S, Liu H B, MacColl R, Zhang X C 2016 Biosens. Bioelectron. 20658

[ 3 ] Penkov N V, Yashin V A, Belosludtsev K N 2021 Appl. Spectrosc. 75189

[ 4 ] Cherkasova O, Nazarov M, Shkurinov A 2016 Opt. Quantum Electron. 48217

[ 5 ] Pickwell E, Wallace V P 2006 Phys. D Appl. Phys. 39 R301

[ 6] Strachan C J, Rades T, Newnham D A, Gordon K C, Pepper M, Taday P F 2004 Chem. Phys. Lett. 39020

[ 7 ] Brown E R, Bjarnason J E, Fedor A M, Korter T M 2007 Appl. Phys. Lett. 90 061908

[ 8 ] Mclntosh A I, Yang B, Goldup S M, Watkinson M, Donnan R S 2013 Chem. Phys. Lett. 558104

[9] Huang R R, Zhao GZ, Liu Y, Kou K, Gu C 2015 Acta Opt. Sin. 35 s230001 （in Chinese) 1黄瑞瑞，赵国忠，刘影，寇宽，顾畅 2015 光学学报 35 s230001]

[10] Lu W L, Lou S Q, Wang X, Shen Y, Sheng X Z 2015 Acta Phys. Sin. 64 114206 （in Chinese）[鹿文亮, 娄淑琴, 王金金, 申艳, 盛新志 2015 物理学报 64 114206]

[11] Chen T, Cai Z H, Hu F R, Yin X H, Xu C P 2019 Spectrosc. Spect. Anal. 39 686（in Chinese） [陈涛，蔡治华，胡放荣，殷贤华，许川佩 2019 光谱 学与光谱分析 39 686] 
[ 12] Wang Y M, Zhao Z S, Qin J Y, Liu H, Liu A F, Xu M M 2020 Talanta 208 120469

[ 13 ] Yang Y H, Shutler A, Grischkowsky D 2011 Opt. Express 198830

[ 14 ] Grognot M, Gallot G 2015 Appl. Phys. Lett. 107103702

[ 15] Shih K L, Pitchappa P, Jin L, Chen C H, Singh R, Lee C 2018 Appl. Phys. Lett. 113071105

[ 16 ] Keshavarz A, Vafapour Z 2019 IEEE Sens. J. 195161

[ 17 ] Kindt J T, Schmuttenmaer C A 1996 J. Phys. Chem.10010373

[ 18 ] Yamauchi S, Hatakeyama S, Imai Y, Tonouchi M2014 Opt. Eng. 53031203

[19] The Cambridge Crystallographic Data Centre https:/www.ccdc.cam.ac.uk/structures/ [2021-11-8]

[ 20 ] Vallet V, Macak P, Wahlgren U, Grenthe I 2006 Theor. Chem. Acc. 115145

[21] Zheng Y Y, Ren G M, Chen R, Wang X M, Chen X H, Wang L, Yuan L, Huang X F 2014 Acta Phys. Sin. 63213101 (in Chinese) [郑圆圆, 任桂明, 陈 锐, 王兴明, 谌晓洪, 主玲, 袁丽, 黄晓凤 2014 物理学报 63 213101]

[22] Takahashi M 2014 Crystals 474

[ 23 ] Grimme S 2004 Comput. Chem. 251463

[24 ] Yu B, Zeng F, Yang Y, Xing Q, Chechin A, Xin X, Zeylikovich I, Alfano R R 2004 Biophys. J. 86164 


\title{
Experimental study and simulation analysis of
} Terahertz absorption spectra of $\alpha$-lactose aqueous solution*

\author{
Hou lei Wang Jun-Nan Wang lei Shi Wei ${ }^{\dagger}$ \\ (Department of Physics, Xi'an University of Technology, Shaanxi 710048, China)
}

Abstract

Water has strong absorption of terahertz $(\mathrm{THz})$ wave, so it has always been a difficult problem to study the characteristics of aqueous samples by $\mathrm{THz}$ technology. In this paper, THz waves with high field strength are obtained in the position of sample by using photoconductive antenna working in high-gain mode and horn shaped graded parallel plate waveguide in $\mathrm{THz}$ time domain spectroscopy system, and the direct detection of $\mathrm{THz}$ spectrum of $\alpha$-lactose solution in the range of $0.1-1.5$ THz was realized. Furthermore, the absorption spectrum of $\alpha$-lactose single molecule model in water environment is simulated by density functional theory, and the simulation results are in good agreement with the experimental results. This work has important reference value for the direct detection of the spectral characteristics of water samples in $\mathrm{THz}$ band.

Keyword: Terahertz waves; Terahertz time-domain spectroscopy system; Density functional theory; $\alpha$-lactose 
This work is supported by the National Natural Science Foundation of China (Grant N os. 62075179, 61575161); the Natural Science Foundation of Shaanxi Province (Grant No. 2019JZ04); the Shaanxi Key Laboratory of Ultrafast Photoelectronic Technology and Terahertz Science (Grant No. 2018SZS06); the Xi'an Key Laboratory of Ultrafast Photoelectronic Device Technology (Grant No. 201805055ZD6CG39).

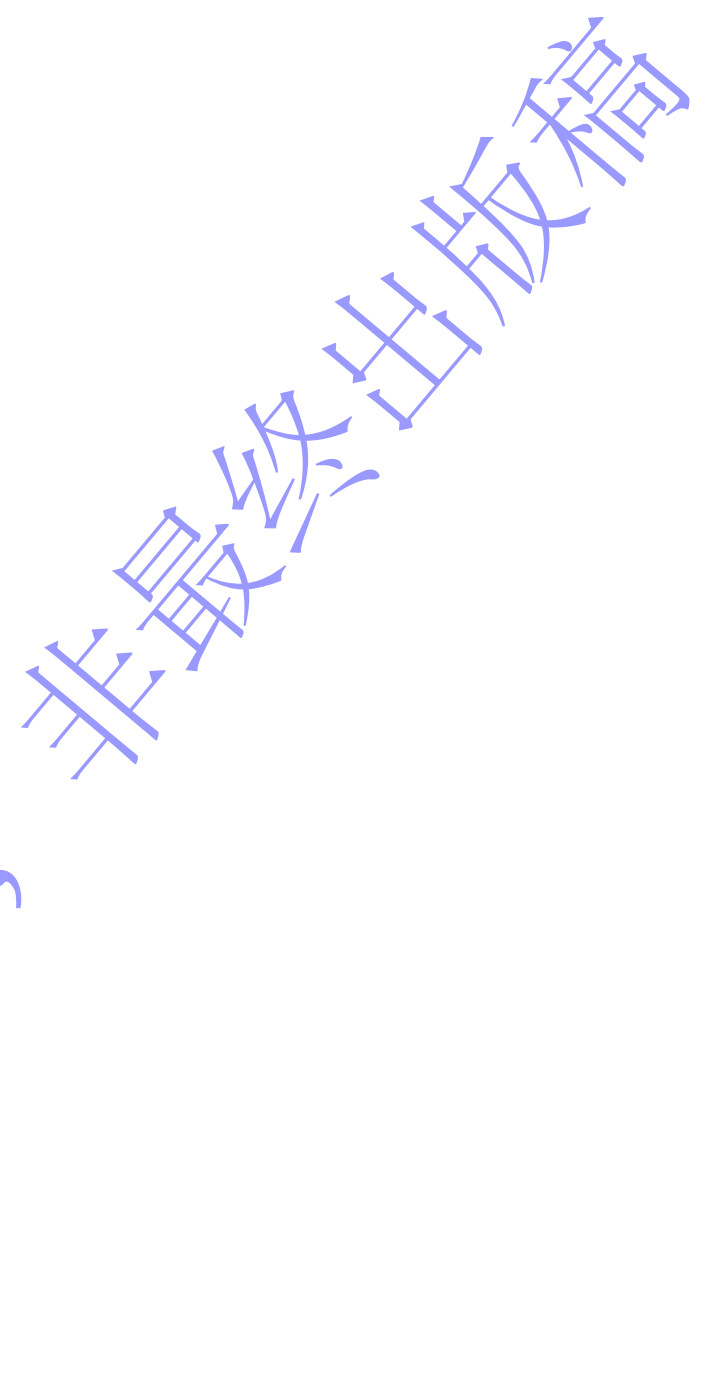

